- Streptomyceten per Anhalter unterwegs

- Testung auf APOE-Allel für Alzheimer-Erkrankung?

- HDAC1 als Schlüsselgen für die Genreparatur

- Budesonid als Therapieoption gegen COVID-19?

DOI: $10.1007 / \mathrm{s} 12268-021-1602-8$

(C) Springer-Verlag GmbH 2021

\section{Mikroorganismus in den Schlagzeilen \\ Streptomyceten per Anhalter unterwegs}

Streptomyceten besitzen keinen Fortbewegungs- und Chemotaxisapparat. Lange galt, dass sie zur Eroberung von neuen Lebensräumen in ihrem natürlichen Habitat Boden auf Wind und Wetter angewiesen sind. Alise Muok et al. (ISME J (2021), doi.org/10.1038/s41396-021-00952-8) zeigen, dass sie sich an Flagellen anderer Mikroorganismen anheften können, um so als „Anhalter“ zu reisen.

- Im Laufe ihres Lebenszyklus verwandeln sich Streptomyceten von filamentösen $\mathrm{Hy}$ phen zu einzelligen Sporen. Während einige verwandte Aktinobakterien, wie Actinoplanes missouriensis, mit Flagellen ausgestattete Sporen ausbilden, fehlen Streptomyceten Strukturen zur aktiven Fortbewegung. Anstatt viel Energie in die Synthese eines eigenen Bewegungsapparats zu stecken, nutzen sie lieber die Flagellen von beweglichen Mitbewohnern wie Bacillus subtilis und Pseudomonas fluorescens und profitieren auch vom chemotaktischen System dieser Bakterien. Muok et al. zeigen, dass schwärmende Bacilli, also Bakterien, die zur Flagellen-vermittelnden kollektiven Fortbewegung auf Oberflächen in der Lage sind, Streptomyceten-Sporen besonders effektiv über mehrere Zentimeter lange Strecken verteilen können. Zur Anheftung von Streptomyceten an die Flagellen von $B$. subtilis scheinen spezielle Oberflächenproteine - die Rodline RdIA und die RdIB - besonders wichtig zu sein.

$\rightarrow$ Die beschriebene "Tramper"-Mobilitätsstrategie ist nicht nur auf Streptomyceten beschränkt. So nutzt auch der unbewegliche Pilz Aspergillus fumigatus den schwärmenden Paenibacillus vortex zur Verteilung seiner Sporen. Wie verbreitet und effektiv diese Strategie in natürlicher Umgebung ist, bleibt allerdings offen. Während der Vorteil für den mitfahrenden Gast auf der Hand liegt, ist der Gewinn einer solchen Interaktion für den mobilen Partner rätselhaft. Wie sich Bacilli mit

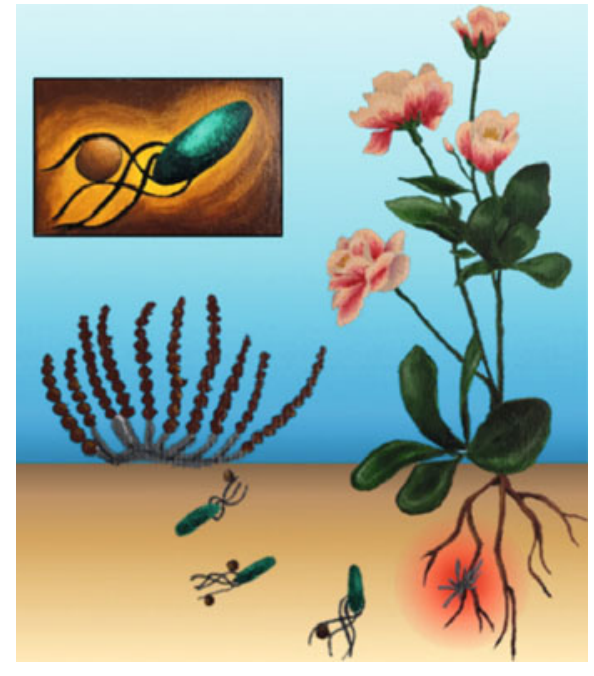

Abb.: Luftgetragene Streptomyces-Sporen werden zu Pflanzenwurzelsystemen transportiert, indem sie direkt an den Geißeln beweglicher Bakterien haften (kleines Bild). Hier keimen die Sporen aus und produzieren Antibiotika (roter Verlauf), um mikrobielle Konkurrenten abzuwehren. Bild aus Muok A et al., ISME J (2021), doi. org/10.1038/s41396-021-00952-8, CC BY 4.0.

solchem Gepäck an den Flagellen überhaupt effektiv bewegen können, bedarf auch weiterer Erforschung.

Natalia Tschowri, Hannover

\title{
Gene in den Schlagzeilen
}

\section{Testung auf APOE-Allel für Alzheimer-Erkrankung?}

Das menschliche Gen für das Apoliporotein $\mathrm{E}(A P O E)$ liegt auf dem Chromosom 19 und zeigt in den Codons 112 und 158 je einen Cys/Arg-Polymorphismus. Entsprechend gibt es mehrere Kombinationsmöglichkeiten: Cys 112/Cys 158 (ApoE2), Cys 112/Arg 158 (ApoE3) und Arg 112/Arg 112 (ApoE4). Diese Allele können natürlich auch hetero- bzw. homozygot vorliegen. Interessanterweise erhöht das homozygote ApoE4-Allel das Risiko, an der späten Form von Alzheimer zu erkranken, um das 15-fache. Oder 91 Prozent der homozygoten ApoE4-Träger erkranken an Alzheimer (umgekehrt hat das ApoE2-Allel eine starke Schutzwirkung).
Das Team um Jalayne Arias von der Universität von Kalifornien in San Francisco ist nun der Frage nachgegangen, ob die acht größten privaten Krankenversicherungsträger in den USA einen entsprechenden genetischen Test bezahlen würden (Arias JJ et al., Genet Med (2021) 23:614-620). Erwartungsgemäß lehnen es die meisten Versicherungsträger $a b$, diesen Test zu bezahlen. Die Begründungen dazu orientieren sich u. a. am Fehlen entsprechender Richtlinien bzw. eines therapeutischen Vorteils für die Patienten oder an einer unzureichenden Datengrundlage.

$\rightarrow$ Unter den gegebenen Umständen ist der Grundsatz der Versicherungen zunächst verständlich, allerdings erscheint eine zukünftige Behandlung von Alzheimer-Patienten mit monoklonalen Antikörpern gegen das
Amyloid- $\beta$-Protein vielversprechend (für eine Metaanalyse siehe Avgerinos KI et al., Ageing Res Rev (2021) 68:101339). Insofern zeichnet sich eine erste Perspektive zur Behandlung der Alzheimer'schen Erkrankung ab - und da wäre es natürlich schon wünschenswert, die potenziellen Patienten mit dem höchsten Risiko identifizieren zu können, wenn die Präparate zugelassen sind. Andererseits gibt es natürlich die direkt an Patienten gerichteten Testverfahren, die beispielsweise von OnlineAnbietern wie „23andMe“ angeboten werden. Dieser Markt wächst stark, und es bleibt zu überlegen, ob Tests auf die ApoE-Allele in Deutschland durch die Krankenkassen bezahlt werden sollten, um so eine hochwertige genetische Beratung sicherzustellen und diese nicht Online-Firmen zu überlassen.

Jochen Graw, Unterschleißheim 


\section{HDAC1 als Schlüsselgen für die Genreparatur}

An der Ausbildung von neurodegenerativen Erkrankungen sind mitunter altersbedingte Anhäufungen von genetischen Schäden beteiligt. Dies wird vor alledem durch Herunterregulation und Verlust von an der Reparatur beteiligten Genen begünstigt. In speziellen Mausmodellen konnte bereits ein Zusammenhang zwischen vermehrten DNA-Läsionen durch 8-Oxoguanin (8-oxoG) nach einem Knockout des Reparaturgens HDAC1 festgestellt werden.

- Im Alter nehmen genetische Schäden zu, während die Reparaturmechanismen schlechter werden. Diese führen zu neurologischen Erkrankungen und einer abnehmenden kognitiven Leistung. Bisher ist nicht viel über die genetischen Mechanismen der DNA-Reparatur bekannt. Man weiß, dass eine Akkumulation von 8-oxoG (8-Oxoguanin) im Gehirn bei Alzheimer und anderen neurodegenerativen Erkrankungen auftritt. Das Gen HDAC1, das an der Reparatur von Doppelstrangbrüchen in Neuronen beteiligt ist, könnte als Kandidat für Reparaturen in alternden Gehirnen gelten.

Um die Bedeutung von HDAC1 für die Reparaturmechanismen herauszufinden, erstell-

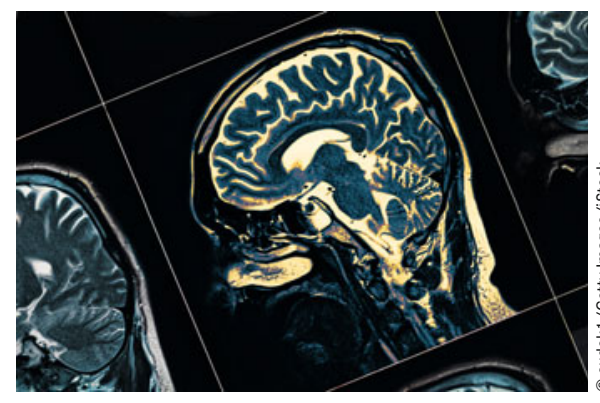

ten P. C. Pao et al. (Nat Commun (2020) 11:2484 ) zunächst eine Hdac1 cKO-Mauslinie, in der HDAC1 in Neuronen und Astrozyten ausgeknockt wurde. Während Jungtiere keine morphologischen Unterschiede zum Wildtypen aufwiesen, wurde in älteren $\mathrm{Hdac} 1$ cKO-Mäusen (> 13 Monate) eine höhere Anzahl an Astrozyten sowie eine stärkere Immunoreaktivität in Gliazellen festgestellt. Gleichzeitig haben diese Mäuse ein schlechteres Erinnerungsvermögen und Lernverhalten.

Durch anschließende ChIP-Sequenzierung und RNA-Sequenzierung von Hippocampusgewebe der älteren Hdac 1 cKO-Mäuse zeigte sich ein verändertes Expressionsmuster diverser Gene. Die von 8-oxoG verursachten oxida- tiven Läsionen an der DNA werden hiermit in Verbindung gebracht. Gleichzeitig ist die Aktivität von OGG 1 vermindert. Eine Kreuzung zwischen Hdac 1 cKO-Mäusen und 5XFADMäusen, die als Alzheimermodell dienen, weist wie erwartet neben einem ähnlichen Regulationsmuster der Genexpression eine noch stärkere Schädigung des Hippocampus durch DNA-Läsionen auf. Wird die Expression von Hdac 1 pharmakologisch erhöht, führt dies sowohl in älteren Wildtypmäusen als auch in 5XFAD-Mäusen zu einer Reduktion der 8-oxoG-vermittelten Läsionen. 5XFAD-Mäuse profitierten zusätzlich von einer verbesserten kognitiven Leistung.

$\rightarrow$ Das Reparaturgen HDAC1 scheint nachweislich eine Rolle beim Schutz vor der altersund krankheitsbedingten Zunahme von genetischen Schäden zu spielen. Insbesondere für das Verhindern oxidativer Schäden durch Akkumulation von 8-oxoG im Gehirn ist HDAC1 relevant. Durch seine Funktion könnte HDAC1 daher zukünftig als Ziel für therapeutische Ansätze zur Behandlung von Alzheimer und weiteren neurodegenerativen Erkrankungen genutzt werden.

Daniela Kruck, Hannover

\section{Arzneimittel und Toxine in den Schlagzeilen Budesonid als Therapieoption gegen COVID-19?}

Vorschläge zur Therapie von COVID-19 schießen derzeit wie Pilze aus dem Boden. Beinahe im Wochentakt werden in den Medien neue Ideen zu Behandlungsmöglichkeiten diskutiert, wobei sich bei näherem Hinsehen meistens ergibt, dass die wissenschaftliche Evidenz relativ gering ist. In aller Regel basieren derartige Berichte auf in silico-Screenings oder in vitro-Versuchen, ohne dass die selbstverständlich notwendigen klinischen Studien durchgeführt wurden. Aus diesem Grundrauschen an Behandlungsvorschlägen stach in letzter Zeit die Meldung hervor, dass Budesonid den Krankheitsverlauf nach eine SARS-CoV-2-Infektion positiv beeinflussen könnte.

- Budesonid gehört zu den Glucocorticoiden und wirkt wie andere Vertreter dieser Gruppe entzündungshemmend und immunsuppressiv. Es ist kostengünstig, relativ weit verbreitet und verfügbar, auch deswegen erhalten vermutlich die aktuellen Meldungen zu einem Einsatz gegen COVID-19 so viel Aufmerksamkeit. Üblicherweise wird Budesonid gegen Erkrankungen wie Asthma bronchiale, COPD, Morbus Crohn oder Colitis ulcerosa eingesetzt, nun wurde es auch als Therapieoption gegen COVID-19 untersucht (Ramakrishnan S et al., Lancet Respir Med (2021), https://doi.org/10.1016/S22132600(21)00160-0).

Hierzu wurde eine offene, randomisierte, kontrollierte Studie durchgeführt, bei der 146 Patienten innerhalb von sieben Tagen nach dem Beginn von COVID-19-Symptomen eine reguläre Therapie jeweils mit oder ohne Budesonid erhalten hatten. Primärer Endpunkt war ein schwerer Verlauf, der zur Vorstellung in der Notaufnahme bzw. Hospitalisierung führte. Dies trat bei 2 von 73 (3\%) Patienten in der Budesonidgruppe und bei 11 von 73 (15\%) Patienten in der Kontrollgruppe auf, es war also ein deutlicher Unterschied zu beobachten. Auch sekundäre Endpunkte wie Symptomverlauf, Fieber, u. a. wurden positiv beeinflusst. Es traten keine relevanten unerwünschten Wirkungen auf.

$\rightarrow$ Die Ergebnisse dieser Studie sind ermutigend und zeigen, dass sich weitere Untersuchungen lohnen. Allerdings kann auf Basis dieser Daten noch keine allgemeingültige Empfehlung zur Anwendung von Budesonid ausgesprochen werden, denn die Befunde sind mit relativ hohen Unsicherheiten verbunden, insbesondere aufgrund der fehlenden Verblindung und der geringen Patientenzahlen. OffLabel-Verordnungen erscheinen daher zum jetzigen Zeitpunkt nicht sinnvoll. Eine Zunahme der Verordnungen konnte jedoch in letzter Zeit festgestellt werden, in Österreich kam es teilweise sogar zu Versorgungsengpässen. Für eine breite Anwendung sollten aber weitere Studien mit höherem Evidenzgrad abgewartet werden.

Henning Hintzsche, Erlangen, Würzburg 